TITLE:

\title{
Muscle stiffness of posterior lower leg in runners with a history of medial tibial stress syndrome
}

AUTHOR(S):

Saeki, J.; Nakamura, M.; Nakao, S.; Fujita, K.;

Yanase, K.; Ichihashi, N.

\section{CITATION:}

Saeki, J....[et al]. Muscle stiffness of posterior lower leg in runners with a history of medial tibial stress syndrome. Scandinavian journal of medicine \& science in sports 2018, 28(1): 246-251

\section{ISSUE DATE:}

2018-01

URL:

http://hdl.handle.net/2433/231407

\section{RIGHT:}

This is the accepted version of the following article: [J. Saeki M. Nakamura S. Nakao K. Fuijta K. Yanase N. Ichihashi. Muscle stiffness of posterior lower leg in runners with a history of medial tibial stress syndrome. Scandinavian journal of medicine \& science in sports, 28(1), 246-251], which has been published in final form at [https://doi.org/10.1038/s41467018-04449-17. This article may be used for non-commercial purposes in accordance with Wiley Terms and Conditions for Self-Archiving. The full-text file will be made open to the public on 16 January 2019 in accordance with publisher's 'Terms and Conditions for Self-Archiving', この論文は出版社版でありません。引用の際には出版社版をご確認ご利用 ください。; This is not the published version. Please cite only the published version. 
Muscle stiffness of posterior lower leg in runners with a history of medial tibial stress syndrome

\section{Running title: Shear modulus in runners with MTSS history}

Junya Saeki $^{\mathrm{a}, \mathrm{b}}$, Masatoshi Nakamurac ${ }^{\mathrm{c}}$, Sayaka Nakao ${ }^{\mathrm{a}}$, Kosuke Fujita ${ }^{\mathrm{d}}$, Ko Yanase ${ }^{\mathrm{a}}$, Noriaki Ichihashi ${ }^{\mathrm{a}}$

${ }^{a}$ Human Health Sciences, Graduate School of Medicine, Kyoto University, 53 Shogoin-Kawahara-cho,

Sakyo-ku, Kyoto 606-8507, Japan

${ }^{b}$ Research Fellow of the Japan Society for the Promotion of Science, 5-3-1 Kojimachi, Chiyoda-ku,

Tokyo 102-0083, Japan

'Institute for Human Movement and Medical Sciences, Niigata University of Health and Welfare, 1398

Shimami-cho, Kita-ku, Niigata 950-3198, Japan

${ }^{\mathrm{d}}$ Department of Physical Therapy, Nagoya University Hospital, 65 Tsurumai-cho, Syowa-ku, Nagoya

466-0065, Japan

*Corresponding author: Junya Saeki

Human Health Sciences, Graduate School of Medicine, Kyoto University

53 Shogoin-Kawahara-cho, Sakyo-ku, Kyoto 606-8507, Japan

Telephone: +81-75-751-3935; Fax: +81-75-751-3909

E-mail: saeki.junya.55z@st.kyoto-u.ac.jp 


\section{Abstract}

Previous history of medial tibial stress syndrome (MTSS) is a risk factor for MTSS relapse, which suggests that there might be some physical factors that are related to MTSS development in runners with a history of MTSS. The relationship between MTSS and muscle stiffness can be assessed in a cross-sectional study that measures muscle stiffness in subjects with a history of MTSS, who do not have pain at the time of measurement, and in those without a history of MTSS. The purpose of this study was to compare the shear elastic modulus, which is an index of muscle stiffness, of all posterior lower leg muscles of subjects with a history of MTSS and those with no history and investigate which muscles could be related to MTSS. Twenty-four male collegiate runners (age, $20.0 \pm 1.7$ years; height, $172.7 \pm 4.8 \mathrm{~cm}$; weight, $57.3 \pm 3.7 \mathrm{~kg}$ ) participated in this study; 14 had a history of MTSS, and 10 did not. The shear elastic moduli of the lateral gastrocnemius, medial gastrocnemius, soleus, peroneus longus, peroneus brevis, flexor hallucis longus, flexor digitorum longus, and tibialis posterior were measured using shear wave elastography. The shear elastic moduli of the flexor digitorum longus and tibialis posterior were significantly higher in subjects with a history of MTSS than in those with no history. However, there was no significant difference in the shear elastic moduli of other muscles. The results of this study suggest that flexor digitorum longus and tibialis posterior stiffness could be related to MTSS. 
Keywords: medial tibial stress syndrome, shin splints, shear-wave elastography, shear elastic modulus,

muscle stiffness, muscle hardness, flexor digitorum longus, tibialis posterior 


\section{Introduction}

Medial tibial stress syndrome (MTSS) is one of the most common exercise-induced leg disorders in runners (Taunton et al. 2002; Moen et al. 2009). Although many studies investigated the effect of interventions to prevent MTSS, the only method with clear evidence of benefit was the use of shockabsorbing insoles (Moen \& Tol 2009). Conversely, it was reported that wearing thick-soled shoes decreased running economy (Fuller et al. 2015). Thus, understanding of the etiology and correct intervention is important for preventing MTSS, without hampering running performance.

A magnetic resonance imaging study showed that MTSS is the result of a lesion at the junction of the periosteum and fascia (Moen \& Tol 2009). A cadaveric study reported that elongational stress on the tendon of the soleus (SOL), flexor digitorum longus (FDL), and tibialis posterior (TP) increases the strain on the tibial fascia (Bouche \& Johnson 2007). Therefore, it is considered that elongational stress on the lower limb muscles could result in MTSS. Furthermore, previous studies reported that the FDL and SOL attach to the posteromedial border of the tibia, which is the most common site affected by MTSS (Edama et al. 2017; Brown 2016); this suggests that MTSS could be caused by the elongational stress on these muscles due to excessive stiffness.

In a previous study that focused on muscle stiffness, muscle stiffness of the lateral gastrocnemius (LG), medial gastrocnemius (MG), SOL, peroneus longus (PL), and tibialis anterior were significantly higher in MTSS patients who had pain at the time of measurement than in healthy subjects (Akiyama 
et al. 2016); however, the higher muscle stiffness observed in MTSS patients could be a result of stiffness caused by pain-induced spasms (Simons \& Mense 1998). Thus, the muscle related to MTSS development cannot be identified by measuring muscle stiffness in subjects who have pain at the time of measurement. Therefore, in order to investigate the relationship between MTSS and muscle stiffness, a cross-sectional study would be required to measure muscle stiffness in subjects who have a history of MTSS and do not have pain at the time of measurement. In addition, the previous study did not measure stiffness of the FDL and TP (Akiyama et al. 2016), which could be related to MTSS development (Bouche \& Johnson 2007). It is important to measure the stiffness of the FDL and TP in addition to muscles reported in the previous study in order to identify which muscle is related to the development of MTSS. Further, studies have reported that previous history of MTSS is one of the risk factors for MTSS relapse (Hubbard et al. 2009; Newman et al. 2013). This suggests that there must be some physical factors related to MTSS development in runners with a history of MTSS. Therefore, a cross-sectional study investigating the relationship between MTSS and muscle stiffness could be useful to determine the risk factors for MTSS.

Therefore, the purpose of this study was to compare the muscle stiffnesses of all posterior lower leg between subjects with a history of MTSS and those with no history and clarify which muscle is related to MTSS development. We hypothesized that muscle stiffness of the FDL, which is attached to the most common site affected by MTSS, is higher in subjects with a history of MTSS than in those 
with no history.

\section{Material and methods}

\section{Subjects}

Twenty-four male collegiate runners (age, $20.0 \pm 1.7$ years; height, $172.7 \pm 4.8 \mathrm{~cm}$; weight, 57.3 $\pm 3.7 \mathrm{~kg}$ ) participated in this study. Subjects were included if they had a history of either bilateral or no MTSS. History of MTSS was defined as follows: (1) experiencing exercise-induced pain on the posteromedial border of the tibia, (2) experiencing pain along or feeling discomfort on palpation of the posteromedial border of the tibia, and (3) experiencing pain over an area of $\geq 5 \mathrm{~cm}$ along the posteromedial border of the tibia. These criteria were obtained from a previous study (Yates \& White 2004). Subjects with a history of lower limb fracture, those who experienced pain in the lower limb while running at the time of intervention, and those who had a history of unilateral MTSS were excluded. Seventy runners were eligible to participate in this study. Of these, 22 declined to participate, 18 failed to meet the MTSS history criteria, and 6 met the exclusion criteria (Fig. 1). Finally, 24 runners were included in the study, of which 14 had a history of bilateral MTSS and 10 had no history of MTSS. Subjects were given precise information about the content of the study, and informed consent was obtained from all subjects. This study was approved by the ethics committee of Kyoto University Graduate School and the Faculty of Medicine (R0266). 
Muscle stiffness was measured using ultrasonic shear wave elastography imaging (Aixplorer; Supersonic Imagine, France). This method is able to measure muscle stiffness quantitatively in vivo as shear elastic modulus, which is calculated from the velocity of the shear wave created by vibration of tissue (Hug et al. 2015). In a cadaver study, there was a strong correlation between muscle elongation and shear elastic modulus measured by shear wave elastography (Eby et al. 2013). Moreover, shear elastic modulus correlates strongly with torque during passive joint movement and force production intensity in vivo (Ates et al. 2015; Maisetti et al. 2012). Thus, shear elastic modulus measured with shear wave elastography is considered an index of the mechanical characteristics (i.e., stiffness) of the muscle (Hug \& Tucker 2015). The shear elastic modulus of the muscles was measured with a linear array probe (SL10-2; Supersonic Imagine, France). The region of interest (ROI) was set near the center of each muscle. By adopting the method of a previous study (Gennisson et al. 2010), the shear elastic modulus $(\mu)$ was calculated using the following formula:

$\mu(\mathrm{kPa})=\rho \mathrm{V}_{\mathrm{s}}^{2}$, where $V_{s}$ is the shear wave velocity, and $\rho$ is the muscle mass density $\left(1000 \mathrm{~kg} / \mathrm{m}^{3}\right)$. The analysis area was a circular region with diameter of $5 \mathrm{~mm}$ in the center of the ROI (Akagi \& Takahashi 2014). The shear elastic modulus of each muscle was measured three times, and the average was used to determine muscle stiffness. The average value obtained for the right and left leg of each subject was used for further analysis. 
The MG, LG, SOL, PL, peroneus brevis (PB), flexor halluces longus (FHL), FDL, and TP were the target muscles. The shear elastic moduli of the LG, MG, SOL, PL, and PB were measured when the subject was in a prone position, while the FDL and TP values were obtained with the subject in a supine position. The specific locations where the shear elastic modulus was measured are shown in Fig. 2. The location of the LG, MG, and SOL was determined with reference to previous studies (Akagi et al. 2015; Nakamura et al. 2014). The location of the PL, PB, FHL, FDL, and TP was identified by confirming movement of muscle fibers in a B-mode ultrasound image during passive movement of the ankle or toes.

The subjects lay on an electrodynamometer (Biodex System 4; Biodex, USA) with the knee in full extension and the ankle joint securely attached to the footplate of the dynamometer. They were instructed to remain relaxed during the measurements. Ultrasound images were recorded along the longitudinal axis of the muscle at an ankle dorsiflexion of $20^{\circ}$ and a metatarsophalangeal joint dorsiflexion of $0^{\circ}$ (Fig. 3). Measurement was performed at intervals of three days or more at record meets or training camps. In addition, the subjects avoided practice before measurement on the day of the study.

\section{Statistical analyses}

Statistical analyses were performed using statistical software (SPSS Statistics 22; IBM, USA). To assess the reliability of the measurements in all subjects ( $n=24$ right legs) in this study, intra-class 
correlation (ICC) (1.1) was determined. In addition, the coefficient of variation (CV) was also assessed.

The CV was calculated by dividing the standard deviation (SD) of repeated measurements by the average value. As described in previous studies (Landis \& Koch 1977), the reliability was defined as almost perfect if the ICC was $>0.81$.

Descriptive data are presented as the mean $\pm \mathrm{SD}$. The unpaired t-test was used to compare the shear elastic modulus between subjects with a history of MTSS and those with no history. For all tests, the statistical significance was set at $\mathrm{p}<0.05$.

\section{Results}

The reliability results are shown in Table 1 . The ICC values for all muscles were higher than 0.81 .

The shear elastic modulus measurements of both subjects with a history of MTSS and those with no MTSS history are shown in Table 2. The shear elastic modulus of the FDL and TP was significantly higher in subjects with a history of MTSS (9.8 $\pm 1.9 \mathrm{kPa}$ for FDL; $12.7 \pm 4.3 \mathrm{kPa}$ for TP) than in those with no history (7.5 $\pm 1.4 \mathrm{kPa}$ for FDL; $9.2 \pm 3.1 \mathrm{kPa}$ for TP) $(\mathrm{p}<0.01$ for FDL; $\mathrm{p}<0.05$ for TP). However, there were no significant differences in the other muscles between subjects with a history of MTSS and those with no history ( $\mathrm{p}>0.05)$.

\section{Discussion}


This study investigated which muscle could be related to MTSS development by comparing the shear elastic modulus of posterior lower limb muscles between subjects with a history of MTSS and those with no history. The shear elastic moduli of the FDL and TP were higher in subjects with a history of MTSS than in those with no history. To the best of our knowledge, this is the first study to compare the muscle stiffness of the FDL, TP, and FHL between runners with and without a history of MTSS.

The shear elastic modulus of the FDL was significantly higher in subjects with a history of MTSS than in those with no history. This result confirmed our hypothesis and supported previous studies that reported that the FDL, which is attached to the site most commonly affected by MTSS, i.e., the posteromedial border of the tibia, is related to MTSS (Edama et al. 2015; Brown 2016). Other studies have reported that a previous history of MTSS is one of the risk factors for MTSS (Hubbard et al. 2009; Newman et al. 2013), which suggests that there must be some physical factors that are related to MTSS development in runners with a history of MTSS. Because muscle stiffness is known to decrease by stretching (Akagi \& Takahashi 2014; Nakamura et al. 2016), stretching of the FDL could aid in preventing an MTSS relapse. In contrast, one study reported that stretching had no significant effect on the prevention of MTSS (Pope et al. 2000). However, the stretching method used in the study by Pope et al. (2000) involved stretching of the gastrocnemius and SOL, and therefore it is unclear whether stretching of the FDL could prevent MTSS development and relapse. Further study is needed to investigate whether selective stretching of the FDL can prevent MTSS. 
Further, the shear elastic modulus of the TP, which is not attached to the site most commonly affected by MTSS (Edama et al. 2017; Brown 2016), was also higher in subjects with a history of MTSS than in subjects with no history. This result was not in line with our hypothesis. The higher shear elastic modulus of the TP could be a result of myofascial force transmission to a neighboring muscle (Yucesoy et al. 2005), which in this case would be to the TP and FDL that is attached to the site most commonly affected by MTSS (Spinner \& Howe 2015). Thus, stiffness of the TP could play a role in MTSS relapse via the fascia acting as an intermediary or the TP stiffness could increase along with the FDL stiffness. Further studies are needed to investigate the interaction between muscles with regard to muscle stiffness.

Except for the stiffness of the FDL and TP, there were no significant differences in the muscles between subjects with a history of MTSS and those with no history. This result conflicts with the result of a previous study, which reported that muscle stiffness of the MG, LG, SOL, and PL was higher in MTSS patients who had pain at the time of measurement than in healthy subjects (Akiyama et al. 2016); however, the subjects had pain at the time of measurement, which was not the case in our study. It has been pointed out that the spasms induced by pain can lead to muscle stiffness (Simons \& Mense 1998). Thus, pain could be a reason for the high muscle stiffness values observed in MTSS patients in the study by Akiyama et al. Further, our study identified the muscles that could be related to MTSS by 
investigating muscle stiffness in subjects with a history of MTSS who did not have pain at the time of measurement.

This study has limitations. First, we could not obtain reliable measurements of shear elastic modulus at locations other than those in this study because of distortion of the shear wave. Whether shear elastic modulus measured at of each muscle location in this study is representative of the entire muscle was unclear. However, it was thought that shear elastic modulus values could be compared because the measured location in each muscle was determined as a percentage to ensure equivalence between subjects. Second, because this study was a cross-sectional study, it is not clear whether an increase in FDL and TP stiffness would induce an MTSS relapse. A prospective study is needed to investigate whether an increase in FDL and TP stiffness could be a risk factor for MTSS development.

\section{Conclusion}

The shear elastic moduli of the FDL and TP were higher in subjects with a history of MTSS than in those with no history of MTSS. This study will support understanding of MTSS.

\section{Perspectives}

Because the main cause underlying the development of MTSS is not known, there is no available literature on MTSS prevention or treatment methods. The results of this study suggest that controlling 
stiffness of the FDL and TP through stretching could play an important role in preventing an MTSS

relapse. 


\section{References}

Akagi R, Takahashi H. Effect of a 5-week static stretching program on hardness of the gastrocnemius muscle. Scand J Med Sci Sports 2014: 24: 950-957.

Akagi R, Yamashita Y, Ueyasu Y. Age-Related Differences in Muscle Shear Moduli in the Lower Extremity. Ultrasound Med Biol. 2015: 41: 2906-2912.

Akiyama K, Akagi R, Hirayama K, Hirose N, Takahashi H, Fukubayshi T. Shear modulus of the lower leg muscles in patients with medial tibial stress syndrome. Ultrasound Med Biol 2016: 42: $1779-1783$.

Ates F, Hug F, Bouillard K, Jubeau M, Frappart T, Couade M, Bercoff J, Nordez A. Muscle shear elastic modulus is linearly related to muscle torque over the entire range of isometric contraction intensity. J Electromyogr Kinesiol. 2015: 25: 703-708.

Bouche RT, Johnson CH. Medial tibial stress syndrome (tibial fasciitis): a proposed pathomechanical model involving fascial traction. J Am Podiatr Med Assoc 2007: 97: 31-36.

Brown AA. Medial tibial stress syndrome: Muscles located at the site of pain. Scientifica (Cairo) 2016: 2016: 7097489.

Eby SF, Song P, Chen S, Chen Q, Greenleaf JF, An KN. Validation of shear wave elastography in skeletal muscle. J Biomech 2013: 46: 2381-2387.

Edama M, Onishi H, Kubo M, Takabayashi T, Yokoyama E, Inai T, Watanabe H, Nashimoto S, Koga 
Y, Kageyama I. Gender differences of muscle and crural fascia origins in relation to the occurrence of medial tibial stress syndrome. Scand J Med Sci Sports 2017: 27: 203-208.

Fuller JT, Bellenger CR, Thewlis D, Tsiros MD, Buckley JD. The effect of footwear on running performance and running economy in distance runners. Sports Med. 2015: 45: 411-422.

Gennisson JL, Deffieux T, Mace E, Montaldo G, Fink M, Tanter M. Viscoelastic and anisotropic mechanical properties of in vivo muscle tissue assessed by supersonic shear imaging. Ultrasound Med Biol 2010: 36: 789-801.

Hubbard TJ, Carpenter EM, Cordova ML. Contributing factors to medial tibial stress syndrome: a prospective investigation. Med Sci Sports Exerc 2009: 41: 490-496.

Hug F, Tucker K, Gennisson JL, Tanter M, Nordez A. Elastography for muscle biomechanics: Toward the estimation of individual muscle force. Exerc Sport Sci Rev 2015: 43: 125-133. Landis JR, Koch GG. The measurement of observer agreement for categorical data. Biometrics 1977: 33: 159-174.

Maisetti O, Hug F, Bouillard K, Nordez A. Characterization of passive elastic properties of the human medial gastrocnemius muscle belly using supersonic shear imaging. J Biomech 2012: 45: 978-984.

Moen MH, Tol JL, Weir A, Steunebrink M, De Winter TC. Medial tibial stress syndrome: a critical review. Sports Med 2009: 39: 523-546. 
Nakamura M, Ikezoe T, Kobayashi T, Umegaki H, Takeno Y, Nishishita S, Ichihashi N. Acute effects of static stretching on muscle hardness of the medial gastrocnemius muscle belly in humans: an ultrasonic shear-wave elastography study. Ultrasound Med Biol. 2014: 40: 1991-1997.

Nakamura M, Ikezoe T, Umegaki H, Kobayashi T, Nishishita S, Ichihashi N. Changes in passive properties of the gastrocnemius muscle-tendon unit during a 4-week routine static stretching program. J Sport Rehabil 2016. (in press)

Newman P, Witchalls J, Waddington G, Adams R. Risk factors associated with medial tibial stress syndrome in runners: a systematic review and meta-analysis. Open Access J Sports Med 2013: 4: 229-241.

Simons DG, Mense S. Understanding and measurement of muscle tone as related to clinical muscle pain. Pain 1998: 75: 1-17.

Spinner RJ, Howe BM. Leg. In: Standring S, ed. Gray's Anatomy: the Anatomical Basis of Clinical Practice. Forty-first ed. Amsterdam: Elsevier, 2015: 1400-1417.

Taunton JE, Ryan MB, Clement DB, McKenzie DC, Lloyd-Smith DR, Zumbo BD. A retrospective case-control analysis of 2002 running injuries. Br J Sports Med 2002: 36: 95-101.

Yates B, White S. The incidence and risk factors in the development of medial tibial stress syndrome among naval recruits. Am J Sports Med 2004: 32: 772-780. 
Yucesoy CA, Baan GC, Koopman BH, Grootenboer HJ, Huijing PA. Pre-strained epimuscular connections cause muscular myofascial force transmission to affect properties of synergistic EHL and EDL muscles of the rat. J Biomech Eng 2005: 127: 819-828. 
Table 1 . Reliability values of the measurements

\begin{tabular}{lllllllll}
\hline & LG & MG & SOL & PL & PB & FHL & FDL & TP \\
\hline CV (\%) & 2.3 & 3.5 & 4.7 & 4.5 & 3.3 & 1.6 & 2.5 & 3.4 \\
& & & & & & & & \\
ICC (1.1) & 0.985 & 0.953 & 0.942 & 0.975 & 0.992 & 0.996 & 0.982 & 0.989 \\
\hline
\end{tabular}

Intra-class correlation (ICC) (1.1) was calculated using three intra-day measurements of the shear elastic modulus in the right limb of all subjects in this study. In addition, the coefficient of variation (CV) was calculated. The CV was calculated by dividing the standard deviation of three repeated measurements by the average of three measurement values.

LG: lateral gastrocnemius; MG: medial gastrocnemius; SOL: soleus; PL: peroneus longus; PB: peroneus brevis; FHL: flexor hallucis longus; FDL: flexor digitorum longus; TP: tibialis posterior 
Table 2. Comparison of the shear elastic modulus between subjects with a history of MTSS and those with no history.

Shear elastic modulus $(\mathrm{kPa})$

\begin{tabular}{cccccc}
\hline & No history of MTSS & History of MTSS & P value & Effect size & Statistical Power \\
\hline LG & $37.0 \pm 8.8$ & $39.7 \pm 8.5$ & 0.457 & $\mathrm{r}=0.160$ & 0.122 \\
MG & $60.9 \pm 8.2$ & $65.9 \pm 11.3$ & 0.572 & $\mathrm{r}=0.122$ & 0.085 \\
SOL & $9.0 \pm 2.6$ & $9.5 \pm 1.8$ & 0.248 & $\mathrm{r}=0.246$ & 0.206 \\
PL & $11.9 \pm 4.7$ & $11.9 \pm 4.3$ & 0.993 & $\mathrm{r}=0.002$ & 0.050 \\
PB & $9.6 \pm 3.4$ & $8.6 \pm 2.2$ & 0.376 & $\mathrm{r}=0.190$ & 0.139 \\
FHL & $8.5 \pm 2.2$ & $9.1 \pm 2.1$ & 0.556 & $\mathrm{r}=0.127$ & 0.088 \\
FDL & $7.5 \pm 1.4$ & $9.8 \pm 1.9$ & 0.004 & $\mathrm{r}=0.561$ & 0.859 \\
TP & $9.2 \pm 3.1$ & $12.7 \pm 4.3$ & 0.036 & $\mathrm{r}=0.431$ & 0.570 \\
\hline
\end{tabular}

MTSS: medial tibial stress syndrome; LG: lateral gastrocnemius; MG: medial gastrocnemius; SOL:

soleus; PL: peroneus longus; PB: peroneus brevis; FHL: flexor hallucis longus; FDL: flexor digitorum longus; TP: tibialis posterior 


\section{Figure Captions}

Fig. 1. Flow diagram depicting subject selection

Fig. 2. Locations of shear elastic modulus measurement

a. Lateral side

The shear elastic moduli of the LG, MG, and SOL were measured at a proximal distance of $30 \%$

of the lower leg length from the popliteal crease to the lateral malleolus. The shear elastic moduli of the PL and PB were measured at a proximal distance of $30 \%$ from the head of the fibula to the lateral malleolus. The shear elastic modulus of the FHL was measured at a proximal distance of $60 \%$ from the head of the fibula to the lateral malleolus.

LG: lateral gastrocnemius; MG: medial gastrocnemius; SOL: soleus; PL: peroneus longus; PB: peroneus brevis; FHL: flexor halluces longus; PC: popliteal crease; FH: head of the fibula; LM: lateral malleolus

b. Medial side

The shear elastic modulus of the FDL was measured at a proximal distance of $50 \%$ from the cleavage line of the knee joint to the medial malleolus. The shear elastic modulus of the TP was measured at a proximal distance of $40 \%$ from the cleavage line of the knee joint to the medial malleolus. 
FDL: flexor digitorum longus; TP: tibialis posterior; CL: cleavage line of the knee joint; MM:

medial malleolus

Fig. 3. Ultrasound images of shear elastic modulus measurement

a: lateral gastrocnemius; b: medial gastrocnemius; c: soleus; d: peroneus longus; e: peroneus brevis;

f: flexor hallucis longus; g: flexor digitorum longus; h: tibialis posterior 
Fig. 1

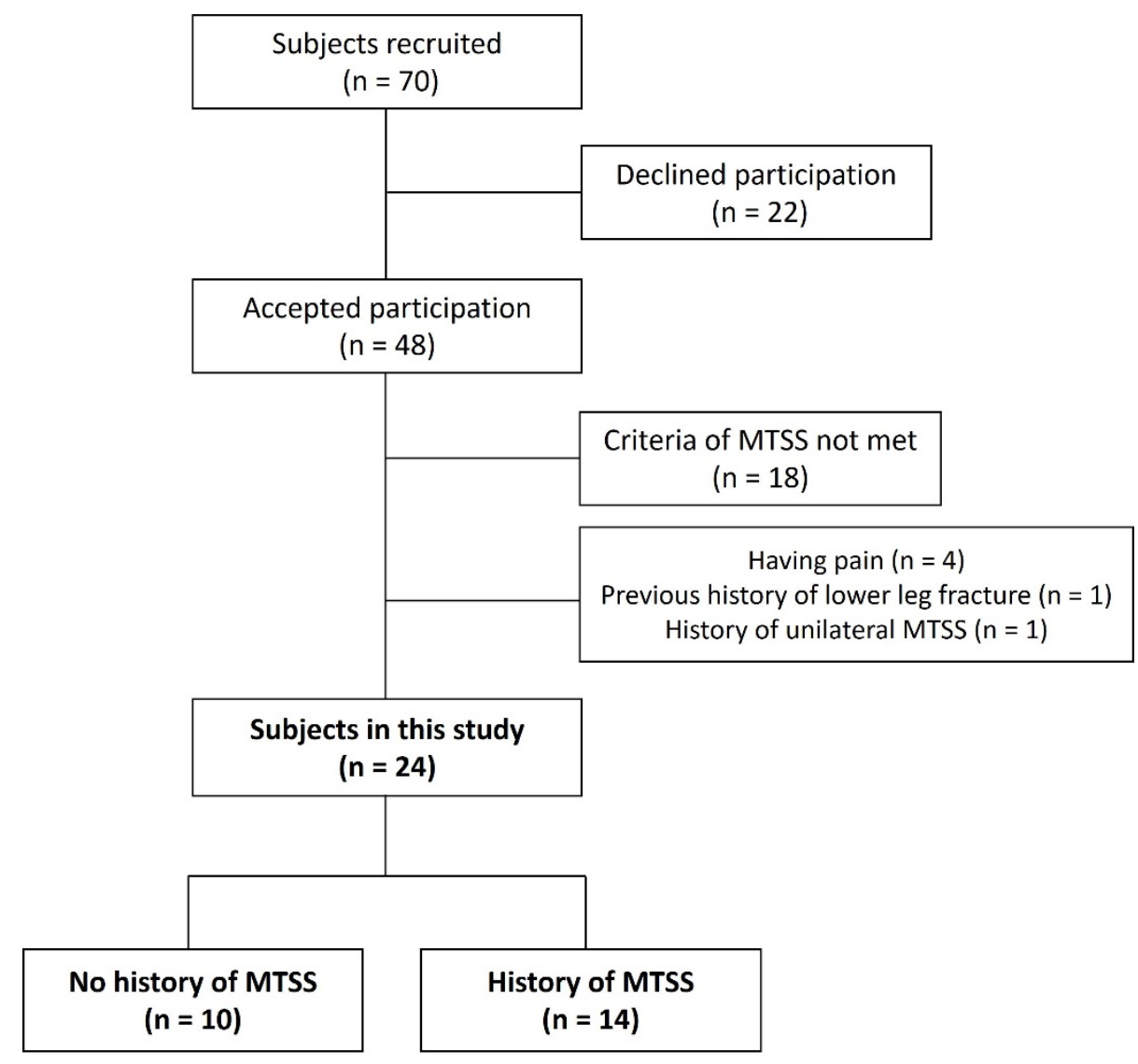


Fig. 2
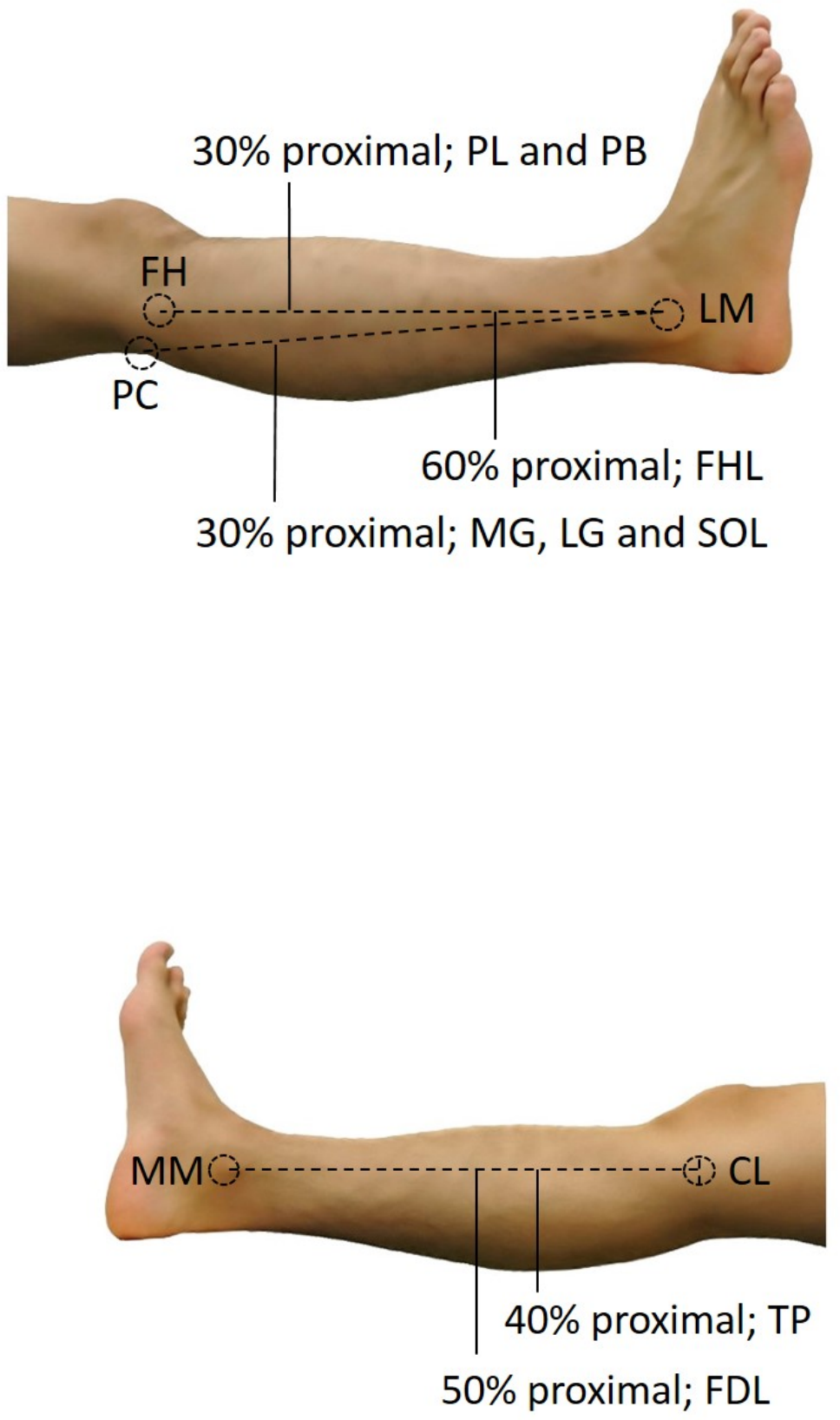
Fig. 3

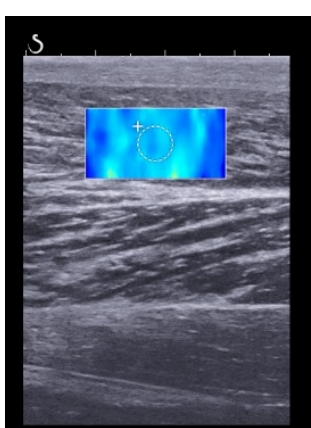

a

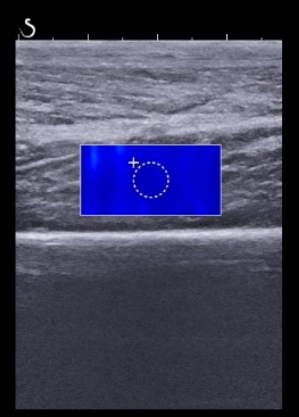

e

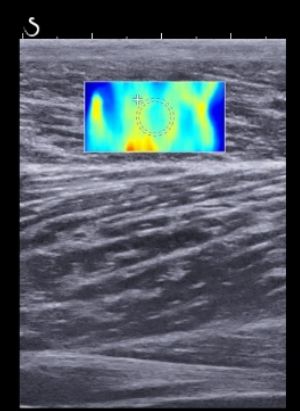

b

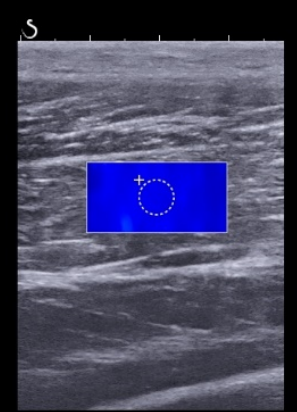

f

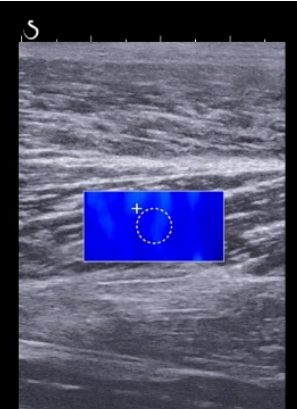

C

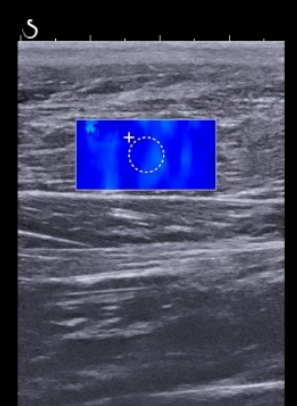

g

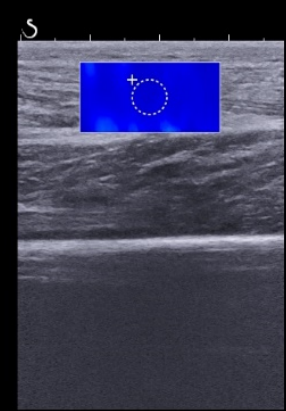

d

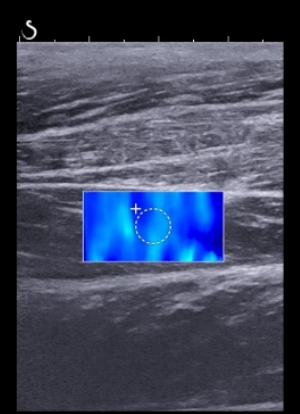

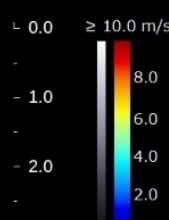

$-3.0$

$-4.0$

$-5.0$ 